\title{
Elaboração e estudo de propriedades psicométricas do Inventário de Práticas Parentais
}

\author{
Silvia Pereira da Cruz Benetti 1 \\ Marcos Alencar Abaide Balbinotti
}

\begin{abstract}
Resumo
O objetivo deste estudo é construir um inventário das práticas de socialização empregadas por pais e mães com filhos na idade escolar. A necessidade de construir e de verificar as qualidades psicométricas desta nova medida das práticas parentais, baseada nas teorias sociocognitivas e sistêmicas, é sistematicamente apresentada. Uma amostra de 106 pais, de ambos os sexos, de crianças com idades que variam entre seis e dez anos foi utilizada para se verificarem as propriedades psicométricas dessa primeira versão. Os resultados obtidos com a amostra em estudo, especificamente aqueles relativos às análises de itens e de dimensões, das correlações e da estrutura fatorial exploratória, corroboram mais fortemente o construto teórico do que as fortes previsões sugeridas por cálculos de correlação interdimensões. Pesquisas são ainda necessárias a fim de prosseguir os estudos psicométricos do IPP.

Palavras-Chave: Práticas parentais; Crianças em idade escolar; Medidas; Validade; Fidedignidade.
\end{abstract}

\section{Elaboration and psychometric evaluation of the Parental Practices Inventory}

\begin{abstract}
The goal of this study is to develop a Parental Practices Inventory (IPP) assessing mothers and fathers' socialization behaviors with school-age children. The need to build and verify the psychometric characteristics of the new instrument, based on social-cognition and systemic theoretical viewpoints, is systematically presented. A sample of 106 parents mothers and fathers with children aged from 6 to 10 years old was utilized to assess the psychometrics characteristics of the first version. Item and dimension analysis, correlation and exploratory factor analysis were performed on the results, which support the theoretical construct of the instrument. Further studies are still needed to obtain more data about the validity of the instrument IPP.

Keywords: Parental practices; School-age children; Measurement; Validity; Reliability.
\end{abstract}

\section{Corte teórico das práticas parentais}

As relações entre pais e filhos e suas influências no desenvolvimento socioemocional e cognitivo de crianças e adolescentes têm sido um dos principais objetos de investigação nas áreas de psicologia do desenvolvimento e clínica (Chen, Liu \& Li, 2000). Desde os trabalhos iniciais de Sears, Maccoby e Levin (1970) sobre como os pais educam seus filhos e quais as conseqüências para o desenvolvimento da criança das variadas formas de socialização, diferentes aspectos das relações parentais foram foco de investigações. Pode-se salientar, por exemplo, as relações pais-filhos e características de apego (Kerns, Aspelmeier, Gentzler \& Grabill, 2001; Rosen \& Rothbaum, 1993; Stein, Williamson, Birmaher, Brent, Kaufman, Dahl, Perel \& Ryan, 2000), as dimensões do estilo parental (Baumrind, 1970; Costa, Teixeira \& Gomes, 2000), a importância das crenças parentais nas práticas educativas (Grusec, Hastings \& Mammone, 1994; Luster \& Okagaki, 1993; Super \& Harkness, 1996), a relação das práticas parentais com a psicopatologia infantil (Wamboldt \& Wamboldt, 2000; Wasserman, Miller, Pinner \& Jaramillo, 1996) e, finalmente, práticas parentais e abuso (Nicholas \& Bieber, 1996).
Atualmente, a importância da relação entre pais e filhos e das práticas de socialização utilizadas para promoção do desenvolvimento psicossocial da criança baseia-se no entendimento de que os processos relacionais entre pais e filhos são situações complexas, que influenciam e são influenciados pelo contexto imediato de inserção da família e por situações mais distais da organização social e histórica da comunidade (Super \& Harkness, 1996; Bronfenbrenner, 1996). Portanto, as práticas de socialização são mediadas e moderadas por diversos fatores, tais como as características psicológicas parentais (Cicchetti \& Toth, 1998; Downey \& Coyne, 1990), a qualidade do relacionamento familiar (Frosch \& Mangelsdorf, 2001; Holden, Geffner \& Jourilles, 1998) e o contexto de inserção das relações familiares (Chao, 1994; Dekovi, Janssens \& Gerris, 1991; Lovejoy, Weis, O’Hare \& Rubin, 1999).

Particularmente, Darling e Steinberg (1993) discriminam três aspectos específicos nas relações entre pais e filhos: os objetivos e valores familiares que orientam os pais no processo de socialização da criança; as práticas parentais efetivamente utilizadas pelos pais para educar a criança; e o estilo parental ou clima emocional no qual a socialização acontece (p. 487). Mais

Endereço para correspondência:

${ }^{1}$ E-mail: silvia@bios.unisinos.br 
precisamente, os objetivos e valores (crenças) que orientam os pais afetariam as práticas parentais (comportamento) e o estilo parental (clima emocional), indicando uma distinção entre a prática e o estilo parental.

Assim, a noção de que o processo de socialização da criança é resultado da interação de diversos níveis ou fatores reflete-se na necessidade das investigações em adotarem abordagens multidimensionais do contexto familiar. $\mathrm{Na}$ área de avaliação e medidas, essa abordagem justifica a necessidade da construção e validação de instrumentos que identifiquem as diferentes dimensões do processo de socialização com critérios adequados de mensuração e sirvam como medidas auxiliares de estudo das características familiares (Lovejoy e colaboradores, 1999). Apesar da existência de instrumentos qualificados ao longo dos anos em suas qualidades psicométricas, já em 1989 Holden e Edwards apontavam que o uso contínuo de instrumentos sem periódicas revalidações ou a utilização de medidas sem clara objetividade quanto ao entendimento do aspecto específico que se desejava avaliar poderia ocasionar problemas de consistência interna e validade de construto.

Dessa forma, o presente artigo trata da elaboração de um breve inventário sobre as características das práticas educativas utilizadas por pais e mães no processo de socialização de crianças entre 612 anos. O objetivo principal é o de desenvolver um inventário sobre as características de envolvimento dos pais no processo de socialização de crianças em idade escolar, explorando os aspectos das práticas parentais no cotidiano da interação com os filhos. Pretende-se elaborar um instrumento de avaliação que possa ser utilizado de forma complementar a outras formas de medidas e que possibilite identificar alguns aspectos do envolvimento parental nesta faixa etária.

Em termos de conteúdo, além dos construtos relacionados à qualidade da relação parental "suporte emocional e envolvimento/hostilidade e coerção" (Arnold, O'Leary, Wolff \& Acker, 1993; Dekovi e colaboradores, 1991; Lovejoy e colaboradores, 1999), considerou-se a questão das características da faixa etária escolhida no processo de socialização da criança e o tipo de envolvimento parental na idade escolar, incluindo características do envolvimento masculino com os filhos. Conforme Lamb, Pleck, Charnov, e Levine (1987), os níveis de envolvimento paterno podem ser: (a) de natureza direta, referindo-se a todas as formas de interação direta com a criança, nas formas de cuidado em geral, brinquedo ou tempo livre com a criança; ou (b) de natureza indireta, acessibilidade ou responsabilidade paterna nas situações em que o pai está presente em casa em atividade que não inclua a participação da criança ou circunstâncias relacionadas ao bem-estar da criança (saúde, escola, e supervisão da criança em geral).

\section{Cortes empíricos das práticas parentais}

Holden (1995) cita a estimativa que, desde o início do século, mais de 100 diferentes instrumentos foram utilizados na avaliação da atitude parental frente ao processo de socialização da criança, baseados no objetivo de identificação das atitudes que melhor beneficiariam o processo de educação da criança. $\mathrm{O}$ conceito de atitude parental utilizado compreende as respostas parentais ante as demandas das diferentes situações enfrentadas no cotidiano relacional com os filhos como, por exemplo, atitudes com relação à disciplina, ao afeto, ao incentivo ao estudo, dentre outras.

Fundamentalmente, a atitude parental é concebida como resultado de três componentes: o cognitivo, o afetivo e o comportamental (Karpinski \& Hilton, 2001). O componente qualitativo-afetivo predomina quando os pais realizam a avaliação dos benefícios e dos aspectos positivos da situação (ou negativos). O componente cognitivo refere-se às crenças ou idéias dos pais sobre a situação. Finalmente, o componente de intenção comportamental diante da situação é a ação propriamente dita, utilizada na interação com a criança (Holden, 1995).

A organização dos instrumentos de avaliação reflete os diversos interesses de investigação como aspectos clínicos, descritivos, teóricos ou metodológicos. $\mathrm{Na}$ grande maioria, a estrutura dos instrumentos é em formato de questionário, tipo Likert, com um número médio de 50 itens ou questões de investigação. Predominam as formas gerais e neutras de descrição de comportamentos infantis, visando à não-indução de respostas dos pais ou sentimentos de culpa diante de itens com atitudes desfavoráveis em relação à criança. A estrutura "Q-sort", onde são oferecidos aos pais a organização das respostas em pilhas de comportamentos, variando entre os mais até os menos descritivos, tem sido citada como a que mais reduz o potencial de viés nas respostas. Entretanto, exige um tempo longo de aplicação e há maior dificuldade na análise e redução dos dados.

Alguns exemplos dos instrumentos mais freqüentemente utilizados nas investigações (Holden, 1995) são o Parental Attitude Research Instrument (PARI), construído por Schaeffer e Bell (1958), o Child Rearing Practices Report (CRPR) de Block, Block \& Morrison, 1981, e o Home Environment Questionnaire (Sines, Clarke \& Lauer, 1984). O Parental Attitude Research Instrument (PARI) é constituído por 23 subescalas (115 itens) que estão organizadas em três fatores: autoritarismo- 
controle, hostilidade-rejeição e democracia-igualitarismo. O segundo instrumento, Child Rearing Practices Report (CRPR), organiza-se originalmente em 91 itens distribuídos em 21 fatores (exemplo: controle, expressão do afeto, preocupações) e é estruturado na forma "Q-sort", a qual permite a distribuição de diversas formas de categorias. Por último, o Home Environment Questionnaire avalia o ambiente psicossocial de crianças entre a quarta e a sexta-série, identificando os fatores ambientais relevantes para o comportamento social e clínico de crianças. É organizado em duas formas (HEQ-1R com 76 itens e HEQ-2R com 134 itens), contendo 10 subescalas: desempenho, agressão-externa, agressão-lar, agressão total, supervisão, mudança, afiliação, separação, sociabilidade e status socioeconômico.

Em termos de qualidades psicométricas, a análise de 83 instrumentos (Holden \& Edwards, 1989) indicou uma média dos índices de consistência de 0,76 (coeficiente de Cronbach) nos diversos estudos de aplicação. Por exemplo, o Child Rearing Practices Report apresentou, em aplicações na cultura original (americana), índices de fidedignidade teste-reteste de 0,71 no intervalo de um (1) ano. O Home Environment Questionnaire apresentou índices de consistência interna, na versão HEQ-2R, adequados para 6 escalas (desempenho, agressão-externa, agressão-lar, agressão total, mudança e nível socioeconômico), variando entre 0,69 a 0,85 , e índices baixos nas escalas separação, afiliação, sociabilidade e supervisão, que variaram de 0,27 a 0,49 (Grotevan \& Carlson, 1989). O Parental Attitude Research Instrument apresentou índices de fidedignidade que variaram de $0,57 \mathrm{a}$ 0,75 na versão materna e 0,75 a 0,87 na versão paterna.

No Brasil, encontram-se referências (Base de Dados Scielo/Lilacs) aos instrumentos Entrevista Familiar Estruturada (EFE), técnica construída e validada para obter os padrões de funcionamento familiar, análise e quantificação das categorias comunicação, regras, papéis de liderança, conflitos, manifestação de agressividade, afeição física, individualização, integração, auto-estima, interação conjugal e interação familiar facilitadora de saúde emocional nos seus membros (Carneiro, 1983). Além desses, há também o Home Environment Questionnaire (HOME) (Bastos, Urpia, Pinho \& Almeida Filho, 1999), a Escala de Responsividade e Exigência (Costa e colaboradores, 2000), e o Parental Attitude Research Instrument (PARI) (Oliveira, Frizzo \& Marin, 2000).

Originalmente adaptado por Nogueira (1988), o PARI obteve índices de fidedignidade teste-reteste (correlação de Pearson) nas subescalas Irritabilidade $r=0,67$; Rejeição $r=0,37$; Intrusão $r=0,39$. Já no estudo de Oliveira, Frizzo e Marin (2000) sobre os efeitos do sexo e da idade da criança pré-escolar nas três atitudes maternas (irritabilidade, rejeição e intrusão), obtiveram-se índices (alpha de Cronbach): Irritabilidade 0,60; Rejeição 0,69; Intrusão 0,78. Na adaptação das Escalas de Responsividade e Exigencia (Costa e colaboradores, 2000) foram encontrados índices de consistência interna (alpha de Cronbach) na escala Responsividade 0,81 e Exigência 0,78.

Quanto ao conteúdo, predomina, nos instrumentos, a análise de mais de uma atitude parental no processo de socialização da criança, sendo a média das subescalas em número de três. Holden cita "atitudes frente à disciplina e controle" e "atitudes relativas ao afeto", como as principais medidas avaliadas (outras atitudes, por exemplo, seriam consistência das práticas educativas, independência vs. dependência). Apesar da diversidade do aspecto ou atitude parental estudada, são freqüentes as investigações sobre atitudes relativas à disciplina, ao afeto, à promoção de autonomia, ao incentivo ao estudo, à aceitação e ao apoio da criança. Nesse sentido, essas categorias refletem os principais construtos encontrados nas pesquisas sobre práticas de socialização, apoio emocional/exigência e hostilidade/ coerção (Lovejoy e colaboradores, 1999).

$\mathrm{O}$ apoio emocional refere-se à responsividade parental, estilo caracterizado por comportamentos que indicam a capacidade dos pais em preocupar-se com o bem-estar da criança, mediante demonstrações de afeto, participação em atividades e oferecimento de suporte emocional e instrumental. Já a dimensão exigência, refere-se ao controle do comportamento em supervisão e estabelecimento de limites. Em oposição às características de responsividade parental, situam-se os comportamentos parentais de hostilidade, baseados em exigências coercitivas e/ou ausência de limites. Essas interações têm sido relacionadas com situaç̧ões familiares de conflitos e estresse (Lovejoy e colaboradores, 1999).

Originalmente proposta por Baumrind (1970), a noção do estilo parental conforme as características autoritárias, autoritativas e permissivas utilizadas na interação com os filhos tem contribuído grandemente para o entendimento do processo de socialização da criança. $\mathrm{O}$ estilo parental autoritário está relacionado com características infantis de imaturidade, insegurança e baixo rendimento escolar. Ao contrário, o estilo parental autoritativo (onde há presença de suporte emocional, exigência, autonomia e comunicação) está associado com a promoção de responsabilidade, cooperação, maturidade psicossocial e sucesso acadêmico.

Entretanto, em certos grupos culturais e étnicos (Chao, 1994), as características do estilo parental autoritário não estavam associadas a baixo desempenho escolar, insegurança e dificuldade de adequação do comportamento. Darling e Steinberg (1993) sugeriram que esta peculiaridade devia-se a um entendimento parcial das práticas de socialização, sendo necessária a 
organização de um modelo de práticas que considerasse tanto os objetivos e valores, orientando os pais no processo de socialização da criança, como as práticas parentais utilizadas pelos pais para educar a criança e, finalmente, o estilo parental ou clima emocional no qual a socialização acontece (Darling \& Steinberg, 1993, p. 487). Mais precisamente, os objetivos e valores que orientam os pais afetariam as práticas parentais e o estilo parental, indicando uma distinção entre ambos. Assim, as inconsistências encontradas nas investigações sobre os estilos parentais, quando características do estilo autoritário dos pais, não foram associadas com comportamentos inadequados; poderiam ser compreendidas ao levar-se em conta a noção de que os objetivos das famílias em diferentes contextos culturais podem ser os mesmos, todavia alcançados de forma diferenciada nas práticas educativas.

\section{Recortes teóricos sobre práticas educativas na idade escolar}

As relações parentais com crianças em idade escolar distinguem-se pelo aspecto de não serem mais exclusivamente determinadas pela necessidade de cuidados básicos de atenção, como exige o recémnascido ou a criança em idade pré-escolar. A criança em idade escolar passa a ter mais capacidade de autonomia e, portanto, maior contato com outros adultos e crianças fora da esfera doméstica, diminuindo o tempo de interação direta com os pais (Collins \& Russell, 1991). Isso, entretanto, não diminui a importância das relações parentais e das práticas educativas utilizadas pelos pais no cuidado e educação das crianças, mas somente reflete uma nova dimensão do mundo social da criança.

Em termos de maturação, a criança entre 6-12 anos passa por um significativo crescimento cognitivo que altera sua capacidade reflexiva do pensamento. Há uma ampliação de seus interesses e da exploração intelectual do mundo, o que requer uma relação qualitativamente mais exigente dos adultos que a cercam, em termos de estimulação e interesse no seu mundo em expansão.

A criança em idade escolar está gradualmente sendo exposta a duas importantes forças socializadoras, quais sejam, a escola e as relações sociais com outros adultos e crianças (Collins \& Russell, 1991; Kerns e colaboradores, 2001). As práticas educativas refletem esses novos domínios sociais e se caracterizam, principalmente, na habilidade dos pais em supervisionar e monitorizar as experiências dos filhos na escola e em perceber a vulnerabilidade da criança a situações de estresse ou conflito em algum aspecto social, dessa forma, acompanhando a expansão do mundo social da criança. A capacidade dos pais de supervisionar e monitorizar a criança tem sido associada ao desenvolvimento de autoconceito positivo, capacidade regulatória e responsabilidade social.

Dessa maneira, observa-se que o tempo de interação direta entre pais-filhos de idade escolar diminui sensivelmente nesta faixa etária. Entretanto, as crianças desta idade ainda citam os pais como as fontes principais de suporte social (Furman \& Buhrmester, 1992), e percebem as figuras parentais como uma fonte de segurança, potencialmente disponíveis quando percebidas como necessárias (Kerns e colaboradores, 2001). Esta característica de menor tempo de contato direto exige maior habilidade dos pais em supervisionar o comportamento da criança, mesmo quando ela não se encontra em convívio direto com eles. Neste sentido, a capacidade de monitorar ou supervisionar a criança está associada à disponibilidade dos pais em ativamente conversar e tentar conhecer suas atividades, a fim de estabelecer objetivos e orientações sobre os acontecimentos aos quais ela está exposta, principalmente as situações estressantes. Além disso, os diálogos introduzem na criança a condição de perceber-se em relação aos outros, fator determinante no desenvolvimento da sua capacidade de empatia com a experiência de colegas e outros adultos. Dessa forma, os pais continuam a exercer um papel fundamental no suporte emocional dos filhos, sendo o objetivo principal do sistema parental a proteção da criança, mediante o reconhecimento por parte dos adultos das situações em que a criança apresenta estados emocionais de desconforto e estresse (Kerns e colaboradores, 2001).

\section{Método}

\section{Sujeitos}

Um total de 106 pais (pai e mãe) de crianças entre 6 e 10 anos de idade concordou (consentimento informado) em participar deste estudo. As famílias foram recrutadas em duas escolas de ensino fundamental e uma creche municipal, todas localizadas na cidade de Porto Alegre, Rio Grande do Sul. Deste total, 58 eram mães $(\mathrm{M}=36,00 ; \mathrm{SD}=0,77$ anos de idade $)$ e 48 eram pais ( $\mathrm{M}=37,44 ; \mathrm{SD}=1,05$ anos de idade). $\mathrm{O}$ nível de escolaridade encontrado foi de 28 pais com segundo grau incompleto (26,4\%), 30 pais com segundo grau completo $(28,3 \%)$ e 48 pais com educação superior $(45,3 \%)$. Quinze pais $(14,2 \%)$ identificaram-se como negros, 83 identificaram-se como brancos $(78,3 \%), 6$ mulatos $(5,7 \%)$ e 1 pai como índio $(0,9 \%)$. Vinte e dois pais $(36,8 \%)$ declararam renda entre 1 e 6 salários mínimos e 82 pais (67\%) declararam renda entre 6 e 10 salários mínimos. A idade média das 58 crianças identificadas no estudo foi de $\mathrm{M}=7,00$ (SD = 1,33), sendo 27 do sexo feminino $(46,6 \%)$ e 31 do sexo masculino $(53,4 \%)$.

Psico-USF, v. 8, n. 2, p. 103-113, Jul./Der. 2003 


\section{Procedimentos}

Tendo em vista todos os aspectos levantados nas questões teóricas e nos instrumentos existentes de investigação antes citados, objetivou-se a construção de um instrumento de avaliação, de fácil aplicação, para investigar as práticas parentais com crianças entre 6-12 anos quanto ao envolvimento ou engajamento na vida da criança. Tomou-se como ponto de partida, em termos de conteúdo, os construtos teóricos fundamentados na literatura, apoio "afetivo/ envolvimento" e "hostilidade/coerção" (Lovejoy e colaboradores, 1999) e as características evolutivas da faixa etária investigada; no nível da criança, a expansão do mundo social com ênfase no aspecto didático e, a nível parental, as características da participação na vida da criança e a habilidade em supervisionar as condutas da criança (Collins \& Russell 1991). Além disso, foram consultados alguns instrumentos de avaliação de práticas parentais, como o Alabama Parenting Questionnaire (Shelton, Frick \& Wooton, 1996) e o Parental Practices Scale (Strayhorn \& Weidman, 1988). Dessa forma, foram criadas quatro dimensões, caracterizando os níveis de envolvimento com a criança nesta faixa etária, incluindo os aspectos de engajamento dos pais em atividades sociais (Social), educativas (Educação), disciplinares (Disciplina) e afetivas (Afeto) com a criança.

\section{Instrumento}

Dois instrumentos foram especialmente construídos para responder os objetivos desta pesquisa. O primeiro deles é chamado Inventário de Práticas Parentais (IPP) e o segundo, Escala de Pertinência e Clareza (EPC), elaborada especialmente para medir o acordo interjuízes. Após a criteriosa construção de 16 itens, respeitando rigorosamente a teoria e apoiando-se no julgamento dos juízes, apresentam-se, a seguir e sistematicamente, os resultados das análises de itens, a matriz de correlação entre as dimensões medidas e a estrutura fatorial exploratória desta primeira versão do IPP.

\section{Construcão dos itens do IPP}

Os diversos itens isolados e a concepção de uma escala total foram derivados da literatura especializada. Benetti e Balbinotti (2002), ao elaborarem o IPP, partiram de um interesse em construir uma escala breve (não mais que 20 itens), não perdendo de vista as qualidades psicométricas deste inventário. Os 16 itens elaborados compreendem quatro dimensões distintas: a primeira se propõe medir o envolvimento afetivo; a segunda, a didática; a terceira, a disciplina; e, finalmente, a quarta mede os aspectos sociais do envolvimento parental (Anexo 1). Cada uma destas quatro dimensões apresenta quatro questões formuladas positivamente. As instruções de respostas à escala são simples, exigindo-se apenas respostas sinceras por parte dos respondentes.

\section{Avaliação dos juízes}

Escala de Pertinência e Clareza (EPC). Este instrumento foi especialmente elaborado (Balbinotti, 2002) para que se possa dar início ao estudo da fidedignidade do IPP pela consistência no julgamento de competentes opiniões especializadas de juízesavaliadores. Ainda, podem-se responder, ao menos em parte, questões relativas à validade de conteúdo através da avaliação positiva dos juízes-avaliadores quanto à pertinência teórica das questões. Para tanto, pensou-se em duas dimensões independentes e específicas: uma medindo a pertinência teórica e a outra, a clareza (na linguagem) dos itens do IPP. Estas duas dimensões são medidas a partir de uma escala bipolar, tipo Lickert, em 5 pontos, partindo de "pouquíssima pertinência (ou clareza)", resposta 1, até "muitíssima pertinência (ou clareza)", resposta 5 .

Para o presente estudo, pediu-se a três professores especialistas (um mestre e dois doutores) de reconhecido saber nesta área em estudo para revisar as 16 questões do IPP, individualmente, considerando a pertinência teórica dos enunciados bem como sua clareza. Salienta-se que o IPP é aplicável a pais (pai e mãe) de crianças com idades variando de 6 a 10 anos. Pelo fato desses juízes não terem sido previamente treinados para responder a EPC, foi somente solicitado que baseassem seu julgamento em sua própria experiência prática e teórica. Espera-se, então, um possível resultado do acordo interjuiz (consistência de suas respostas) baixo, visto que cada decisão foi tomada a partir de um julgamento pessoal e próprio, controlado apenas por sua vivência teórico-prática. Os resultados da consistência no julgamento (acordo interjuiz) calculados resultaram numa correlação intraclasse de $r=$ 0,68 (variação de 0,15 a 0,90 ) para pertinência e de $r=$ 0,41 (variação de $-0,55$ a 0,81 ) para a clareza. Embora os juízes não tenham dado nenhuma nota abaixo de 4 ("muita clareza ou pertinência"), eles pareceram ser mais consistentes entre si, em seu julgamento quanto à pertinência teórica de cada questão. Já quanto à clareza dos itens (considerando a possível pluralidade sociocultural e econômica do público alvo), a consistência em seus julgamentos foi menor.

Mesmo que tenha sido pedido aos juízes que baseassem suas decisões em seus próprios julgamentos teóricos e experiências práticas, a pertinência das questões obteve um resultado satisfatório, o que pode significar que apesar de cada juiz ter um background 
único, eles parecem concordar entre si quanto à pertinência teórica das questões, consideradas entre "muito pertinentes" e "muitíssimo pertinentes" $(M=4,8$, $D P=0,41)$. Quanto à clareza, a consistência dos julgamentos foi baixa como era esperado, embora as questões também tenham sido consideradas de "muito clara" a "muitíssimo clara" $(M=4,8, D P=0,40)$. Esse último resultado pode relacionar-se ao entendimento único que cada juiz acorda ao conceito de clareza ou pode nos fazer levantar a hipótese de que o questionário mereceria um trabalho suplementar para torná-lo com itens ainda mais claros.

\section{Resultados}

\section{Qualidades psicométricas do IPP}

Análise de itens. As correlações item-escala total variaram de 0,06 a 0,57 . Mesmo que possa parecer, num primeiro momento, que, de forma geral, cada um dos 16 itens apresente, individualmente, uma associação linear limítrofe (correlações entre médias e muito fracas - ou inexistentes) com a escala total, estas variações também indicaram a possibilidade de estar sendo medida mais do que uma única dimensão, o que estaria de acordo com os anseios esperados. Assim, realizaram-se cálculos de correlação item-dimensão, respeitando o corte teórico proposto. Dessa forma, as correlações se apresentaram bem mais satisfatórias. As correlações item-dimensão entre os quatro itens da dimensão Afetiva variaram de 0,45 a 0,65. Já, as mesmas correlações concernentes à dimensão Didática variaram de 0,52 a 0,80. Aquelas correlações, referentes à dimensão Disciplina, variaram de 0,29 a 0,40. Finalmente, as correlações item-dimensão para a dimensão Social variaram de 0,22 a 0,43. Pode-se observar que a variabilidade total das correlações item-dimensão foi de 0,22 a 0,80. A Tabela 1 apresenta os dados completos.

Consistência interna. Os coeficientes alfa variaram de 0,55 a 0,82, segundo as quatro dimensões estudadas. A Tabela 1 apresenta esses resultados discriminados por dimensão. Salienta-se que não haverá melhoras nos coeficientes alfa no caso de se retirar algum item de alguma dimensão específica. As únicas duas exceções (os itens Eu vejo TV/escuto música com meu filho (a); Eu converso com ela/ ele sobre religião) afetam muito pouco o valor do alfa. Portanto, devido à complexidade da interpretação de dimensões com diferentes números de itens em uma mesma escala total, optou-se por manter estes dois itens.

Tabela 1 - Análises preliminares da fidedignidade do IPP conforme suas dimensões e itens (N=103)

\begin{tabular}{|c|c|c|c|c|c|c|c|c|c|c|}
\hline \multirow{4}{*}{ Dimensão } & \multirow{4}{*}{ Itens } & \multicolumn{2}{|c|}{$\begin{array}{c}\text { Análises } \\
\text { descritivas }\end{array}$} & \multicolumn{7}{|c|}{ Análises correlacionais } \\
\hline & & \multirow{3}{*}{ Média } & \multirow{3}{*}{ D. P. } & \multirow{3}{*}{$\begin{array}{l}\text { Alfa se } \\
\text { item } \\
\text { retirado }\end{array}$} & \multirow{3}{*}{$\begin{array}{c}\text { Correlação } \\
\text { item-escala } \\
\text { total }\end{array}$} & \multirow{3}{*}{$\begin{array}{c}\text { Correlação } \\
\text { item- } \\
\text { dimensão }\end{array}$} & \multirow{2}{*}{\multicolumn{4}{|c|}{$\begin{array}{c}\text { Intercorrelações } \\
\text { Item-item }\end{array}$}} \\
\hline & & & & & & & & & & \\
\hline & & & & & & & Item 1 & Item 2 & Item 3 & Item 4 \\
\hline \multirow{4}{*}{ Afeto } & 1 & 4,54 & 0,68 & 0,75 & 0,31 & 0,45 & -- & & & \\
\hline & 2 & 4,36 & 0,89 & 0,65 & 0,49 & 0,65 & 0,39 & -- & & \\
\hline & 3 & 4,82 & 0,45 & 0,72 & 0,37 & 0,59 & 0,40 & 0,50 & -- & \\
\hline & 4 & 4,60 & 0,83 & 0,65 & 0,39 & 0,64 & 0,36 & 0,62 & 0,52 & -- \\
\hline \multirow{5}{*}{ Educação } & & & & & & & Item 5 & Item 6 & Item 7 & Item 8 \\
\hline & 5 & 4,36 & 0,93 & 0,78 & 0,47 & 0,64 & -- & & & \\
\hline & 6 & 4,02 & 1,27 & 0,77 & 0,47 & 0,64 & 0,45 & -- & & \\
\hline & 7 & 3,79 & 1,29 & 0,83 & 0,43 & 0,52 & 0,58 & 0,32 & -- & \\
\hline & 8 & 3,98 & 1,26 & 0,69 & 0,57 & 0,79 & 0,57 & 0,82 & 0,48 & -- \\
\hline \multirow{5}{*}{ Disciplina } & & & & & & & Item 9 & Item 10 & Item 11 & Item 12 \\
\hline & 9 & 3,46 & 0,92 & 0,50 & 0,06 & 0,29 & -- & & & \\
\hline & 10 & 2,67 & 1,16 & 0,47 & 0,19 & 0,33 & 0,33 & -- & & \\
\hline & 11 & 2,17 & 1,33 & 0,49 & 0,13 & 0,31 & 0,07 & 0,19 & -- & \\
\hline & 12 & 2,35 & 1,25 & 0,41 & 0,18 & 0,40 & 0,23 & 0,20 & 0,36 & -- \\
\hline \multirow{5}{*}{ Social } & & & & & & & Item 13 & Item 14 & Item 15 & Item 16 \\
\hline & 13 & 3,73 & 1,22 & 0,45 & 0,26 & 0,42 & -- & & & \\
\hline & 14 & 3,43 & 1,34 & 0,44 & 0,38 & 0,43 & 0,39 & -- & & \\
\hline & 15 & 4,18 & 0,97 & 0,60 & 0,23 & 0,21 & 0,18 & 0,13 & -- & \\
\hline & 16 & 2,70 & 1,41 & 0,49 & 0,41 & 0,38 & 0,28 & 0,33 & 0,17 & -- \\
\hline
\end{tabular}


$\mathrm{Na}$ verdade, estes dados apresentam-se, apenas em parte, satisfatórios. Mesmo considerando o fato de que o cálculo alfa é sensível ao número de itens de cada dimensão, as duas com valores inferiores a 0,60 apresentam uma insatisfatória consistência interna que poderá indicar, por um lado, fracas correlações entre os itens dessas duas dimensões e, por outro lado, a necessidade de reconstrução de algumas dessas oito questões.

Matriz de correlações. A Tabela 1 apresenta ainda a matriz de correlações intradimensões. Com base na correlação mediana obtida $(0,34)$, independente da dimensão (que divide a distribuição em duas partes), pode-se concluir que, dos dois grupos de correlações obtidos, apenas um é satisfatório (aquele composto pelas dimensões Afeto e Educação).

Duas constatações podem ser apreciadas a partir desses resultados. Em primeiro lugar, as fracas correlações entre as dimensões Disciplina e Social
$(0,06)$, entre as dimensões Disciplina e Didática $(0,17)$ e, finalmente, entre as dimensões Disciplina e Afeto $(0,11)$ apontam a independência relativa da Disciplina em relação a estas três dimensões. Em segundo lugar, as satisfatórias correlações entre as dimensões Afeto e Didática $(0,40)$, Afeto e Social $(0,32)$ e Didática e Social $(0,39)$ deixam predizer que as análises fatoriais poderão ser testadas em dois fatores, além da verificação em quatro fatores que é exigida pelo corte teórico.

Cálculos de tendência central. A Tabela 2 apresenta um estudo pormenorizado das análises descritivas (tendência central, dispersão e distribuição). Os resultados sugerem uma insatisfatória aderência à distribuição normal, o que era esperado tendo em vista as elevadas modas das dimensões Afeto e Educação e a baixa moda da dimensão Disciplina. A média, a trimédia e a mediana das escalas apresentam resultados relativamente homogêneos.

Tabela 2 - Análises descritivas por dimensão estudada

\begin{tabular}{|c|c|c|c|c|c|c|c|c|}
\hline \multirow[b]{3}{*}{ Dimensões } & & \multicolumn{6}{|c|}{ Tendência Central (Dispersão) } & \multirow[b]{3}{*}{$\mathrm{M}_{\text {od }}$} \\
\hline & & \multicolumn{3}{|c|}{ Por Item } & \multicolumn{3}{|c|}{ Por Escala } & \\
\hline & & $\mathrm{X}$ & Mín & Máx & $\mathrm{X}_{(\mathrm{DP})}$ & $\mathrm{X}_{5 \%}$ & $\mathrm{M}_{\mathrm{ed}}$ & \\
\hline Afeto & 101 & 4,58 & 4,36 & 4,82 & $18,33_{(2,25)}$ & 18,63 & 19,00 & 20,00 \\
\hline Educação & 102 & 4,04 & 3,79 & 4,36 & $16,16_{(3,86)}$ & 16,52 & 17,00 & 20,00 \\
\hline Disciplina & 103 & 2,66 & 2,17 & 3,46 & 10,67 & 10,53 & 10,00 & 9,00 \\
\hline Social & 101 & 3,51 & 2,70 & 4,18 & 14,05 & 14,03 & 14,00 & 16 \\
\hline
\end{tabular}

Validade Fatorial (ou de construto). Antes de proceder às análises fatoriais, sugeridas por um lado pelos cálculos de correlação e, por outro lado, pelo corte teórico, o coeficiente Kaiser-Meyer-Olkin $(\mathrm{KMO}=0,72)$ foi estimado e o teste de esfericidade de Bartlett $(p<0,001)$ foi calculado. Seus respectivos valores indicam que as correlações parciais são adequadas para se proceder ao modelo de análise fatorial. Outros resultados originaram-se dessa análise preliminar. Cinco fatores, tendo uma raiz latente (eigenvalue) superior a um, foram extraídos. Eles explicam $51,4 \%$ da variância. Mesmo que as comunalidades dos itens sejam todas superiores a 0,39 , esta solução fatorial parece pouco satisfatória. Por um lado, encontraram-se itens com saturações fatoriais (intradimensões) superiores a 1,0 e outros inferiores a 0,30 . Por outro lado, foi também encontrada a presença de itens que apresentam saturação fatorial superior a 0,30 em mais de um (1) fator. Além desses dois grupos de aspectos importantes a serem considerados negativamente, acrescenta-se a mistura fatorial originária de itens que saturam predominantemente em uma outra dimensão, diferente daquela que seria teoricamente suposto saturar. Com base nestes resultados preliminares e analisando os resultados Psico-USF, v. 8, n. 2, p. 103-113, Jul./Der. 2003 sugeridos pelo gráfico "scree", realizaram-se outras duas análises fatoriais. Uma restringindo o número de fatores em 2 (como fora previsto pelas correlações) e outra restringindo o número de fatores em 4 (considerando o corte teórico).

Assim, uma análise em componentes principais, seguida de uma rotação varimax serviu para se verificar a estrutura fatorial (dois fatores) do IPP. No caso da estrutura a quatro fatores, optou-se pela análise ULS (considerando a proximidade das correlações estimadas com as variáveis observadas), seguida de uma rotação oblíqua (considerando os índices das correlações parciais entre as dimensões).

Solução a dois fatores. No caso da solução a dois fatores, que explicam $37,7 \%$ da variância, denota-se que apenas um (1) item (adequadamente situado em sua dimensão) apresenta saturação fatorial pouco satisfatória $\left(\mathrm{Sat}_{\mathrm{f}}<0,30\right)$. Neste momento, este pequeno problema não tem agravantes maiores. $\mathrm{Na}$ realidade, como fora predito pelos cálculos de correlação, a resposta fatorial é satisfatória. Não se encontraram itens de um fator, saturando mais fortemente no outro, o que garante a pureza dos dois fatores. Este resultado confirma a independência significativa da dimensão Disciplina $(r=0,05)$. 
Tabela 3 - Soluções fatoriais em dois e quatro fatores para o IPP $(\mathrm{N}=103)$

\begin{tabular}{|c|c|c|c|c|c|c|c|c|c|}
\hline \multirow{3}{*}{ Dimensão } & \multirow{3}{*}{ Itens } & \multicolumn{2}{|c|}{ Comunalidades } & \multicolumn{6}{|c|}{ Matriz de explicação fatorial } \\
\hline & & \multicolumn{2}{|c|}{ Extração em } & \multicolumn{2}{|c|}{ Em 2 fatores } & \multicolumn{4}{|c|}{ Em 4 fatores } \\
\hline & & 2 fatores & 4 fatores & Fator 1 & Fator 2 & Fator 1 & Fator 2 & Fator 3 & Fator 4 \\
\hline \multirow{4}{*}{ Afeto } & 1 & 0,29 & 0,47 & 0,53 & & & 0,49 & & \\
\hline & 2 & 0,50 & 0,69 & 0,71 & & & 0,75 & & \\
\hline & 3 & 0,33 & 0,64 & 0,58 & & & 0,74 & & \\
\hline & 4 & 0,39 & 0,68 & 0,63 & & & 0,77 & & \\
\hline \multirow{4}{*}{ Educação } & 5 & 0,39 & 0,56 & 0,62 & & 0,54 & & & \\
\hline & 6 & 0,43 & 0,61 & 0,65 & & 0,75 & & & \\
\hline & 7 & 0,42 & 0,59 & 0,65 & & 0,47 & & & \\
\hline & 8 & 0,58 & 0,76 & 0,75 & & 0,94 & & & \\
\hline \multirow{5}{*}{ Disciplina } & 9 & 0,40 & 0,45 & & 0,61 & & & 0,42 & \\
\hline & 10 & 0,54 & 0,56 & & 0,73 & & & 0,59 & \\
\hline & 11 & 0,42 & 0,53 & & 0,65 & & & 0,52 & \\
\hline & 12 & 0,51 & 0,55 & $-0,34$ & 0,64 & & & 0,56 & \\
\hline & 13 & 0,11 & 0,57 & 0,34 & & & & & 0,69 \\
\hline \multirow{3}{*}{ Social } & 14 & 0,22 & 0,52 & 0,47 & & & & & 0,53 \\
\hline & 15 & 0,11 & 0,36 & & & & & & 0,30 \\
\hline & 16 & 0,32 & 0,38 & 0,57 & & & & & 0,31 \\
\hline
\end{tabular}

Solução a quatro fatores. No caso da solução a quatro fatores, que explicam $56,2 \%$ da variância, denota-se uma distribuição perfeita dos itens, divididos de acordo com o construto teórico explorado. As saturações fatoriais são satisfatórias. As Tabelas 4 e 5 sintetizam as informações pertinentes das estruturas fatoriais obtidas nas duas análises realizadas (dois e quatro fatores).

Tabela 4 - Resultados alfa e cálculos de distribuição por dimensão estudada

\begin{tabular}{|c|c|c|c|c|c|c|c|c|c|}
\hline \multirow{3}{*}{ Dimensões } & \multirow{3}{*}{$\mathrm{N}$} & \multirow{3}{*}{ Alfa } & \multicolumn{7}{|c|}{ Distribuição } \\
\hline & & & \multicolumn{3}{|c|}{ Normalidade } & \multicolumn{2}{|c|}{ Assimetria } & \multicolumn{2}{|c|}{ Achatamento } \\
\hline & & & $\mathrm{K}-\mathrm{S}$ & $\mathrm{gl}$ & Sig & Skewness & $\mathrm{EP}_{\mathrm{s}}$ & Kurtosis & $\mathrm{EP}_{\mathrm{k}}$ \\
\hline Afeto & 101 & 0,76 & 0,243 & 101 & 0,000 & $-1,929$ & 0,240 & 3,687 & 0,476 \\
\hline Educação & 102 & 0,82 & 0,161 & 102 & 0,000 & $-1,161$ & 0,239 & 0,941 & 0,474 \\
\hline Disciplina & 103 & 0,55 & 0,160 & 103 & 0,000 & 0,660 & 0,238 & $-0,025$ & 0,472 \\
\hline Social & 101 & 0,58 & 0,116 & 101 & 0,002 & 0,096 & 0,240 & $-0,959$ & 0,476 \\
\hline
\end{tabular}

Tabela 5 - Variância total explicada para a matriz fatorial em 2 e 4 fatores

\begin{tabular}{|c|c|c|c|c|c|c|}
\hline \multirow{3}{*}{$\begin{array}{c}\text { Componentes } \\
\text { fatoriais }\end{array}$} & \multicolumn{6}{|c|}{ Saturações da soma dos quadrados rotados } \\
\hline & \multicolumn{3}{|c|}{ Em 2 fatores } & \multicolumn{3}{|c|}{ Em 4 fatores } \\
\hline & Total & $\begin{array}{c}\% \text { de } \\
\text { variância }\end{array}$ & $\begin{array}{c}\% \\
\text { cumulativa }\end{array}$ & Total & \% de variância & $\%$ cumulativa \\
\hline Fator 1 & 4,26 & 26,6 & 26,6 & 4,26 & 26,6 & 26,6 \\
\hline Fator 2 & 1,76 & 11,1 & 37,7 & 1,76 & 11,1 & 37,7 \\
\hline Fator 3 & -- & -- & -- & 1,62 & 10,2 & 47,9 \\
\hline Fator 4 & -- & -- & -- & 1,34 & 8,4 & 56,2 \\
\hline
\end{tabular}


A partir da apresentação desses resultados, obtidos nas análises realizadas, conclui-se que a maior conformidade dos dados está em concordância com o corte teórico proposto. Isso garante a validade de construto do IPP, constituindo-se em um inventário que mede o que se propõe medir.

\section{Discussão}

O objetivo deste estudo foi o de construir e verificar as qualidades psicométricas de uma nova medida de práticas parentais - Inventário de Práticas Parentais (IPP). Este instrumento visa identificar as práticas de socialização de pais de crianças em idade escolar, considerando-se as características evolutivas da faixa etária, em termos de desenvolvimento geral e de socialização; e aspectos da fundamentação teórica do envolvimento parental, referente ao comportamento dos pais baseados em apoio "afetivo/envolvimento" e "hostilidade/coerção" (Lovejoy e colaboradores, 1999). Assim, foram avaliadas quatro dimensões de envolvimento com a criança, incluindo os aspectos de engajamento dos pais em atividades sociais (Social), educativas (Educação), disciplinares (Disciplina) e afetivas (Afeto).

Os resultados gerais das análises psicométricas realizadas mostraram-se satisfatórios. $\mathrm{Na}$ verdade, as únicas exceções foram os resultados alfa das dimensões (disciplina alfa $=0,5467)$ e (social alfa $=0,5808$ ), indicando a possibilidade de melhorias na precisão metrológica de duas das quatro dimensões. Entretanto, salienta-se que a perfeita adequação dos fatores com as dimensões teoricamente propostas deve ser considerada como o principal resultado deste estudo preliminar. Este dado é corroborado pelos resultados do minucioso estudo das correlações, onde se ressalta, mais uma vez, a não-melhoria do alfa caso algum item fosse retirado, as adequadas correlações item-escala total e itemdimensão e, por fim, adequados valores numéricos de intercorrelações item-item (na maioria dos casos) e dimensão-dimensão, não esquecendo, é claro, a independência relativa da dimensão Disciplina.

Dessa forma, a organização dos fatores refletiu os achados teóricos de outros instrumentos nos quais a noção do construto "suporte emocional/hostilidade" e "coerção" caracterizam a base fundamental das relações familiares. Isto é, todos os comportamentos parentais e expressões afetivas organizam-se em ações de investimento afetivo e cuidado dos filhos, contrastando-se às situações, envolvendo comportamentos ou práticas mais hostis e autoritárias. Esse construto refletiu-se adequadamente na análise de dois fatores, onde a dimensão disciplina claramente distinguiu-se das demais.
Já, na análise, os quatro fatores indicaram que as dimensões: Social, Afetiva, Disciplina e Educação dão conta de práticas de engajamento mais específicas que discriminam as ações parentais em termos de atividades/disponibilidade nas relações com os filhos. Dessa forma, considera-se que o instrumento apresenta qualidades métricas para a discriminação de situações mais específicas do envolvimento parental.

Em futuras pesquisas, seria oportuno acordar uma atenção particular no estudo da consistência interna das dimensões Disciplina e Social. Sugere-se a elaboração e a experimentação de novos itens que substituam aqueles de baixa correlação item-dimensão. Pensa-se que os itens: "gritar com filho(a) quando ela/ele faz alguma coisa errada" e "as conversas com meu filho(a) terminam em discussões" da dimensão Disciplina e o item "ver TV/escutar música com meu filho" da dimensão Social devam ser substituídos em novos estudos.

Em suma, considera-se que o Inventário de Práticas Parentais (IPP) é um instrumento que permite identificar as práticas de socialização de crianças em idade escolar, em relação à qualidade do envolvimento dos pais - "suporte emocional/hostilidade" e "coerção". Além disso, o instrumento também discriminou as dimensões específicas das práticas de socialização na faixa etária da criança em idade escolar, considerando as características do desenvolvimento psicológico em geral nas áreas social, didática, afetiva e disciplinar. Dessa forma, a utilização do inventário pode auxiliar no desenvolvimento de pesquisas sobre as características familiares no contexto brasileiro, contribuindo para o aprimoramento de trabalhos na área da psicologia.

\section{Referências}

Arnold, D. S., O’Leary, S. G., Wolff, L. S. \& Acker, M. M. (1993). The Parenting Scale. A measure of dysfunctional parenting in discipline situations. Psychological Assessment, 5 (2), 137-144.

Balbinotti, M. A. A. (2002). Escala de Pertinência e Clareza. Manuscrito não publicado. São Leopoldo, RS: Unisinos, Centro de Ciências da Saúde.

Bastos, A. C. S., Urpia, A. C. M., Pinho, L. \& Almeida Filho, N. M. (1999). O impacto do ambiente familiar nos primeiros anos de vida: um estudo com adolescentes de uma invasão de Salvador, Bahia. Estudos de Psicologia,4 (2), 239-271.

Baumrind, D. (1970). Parental control and parental love. Em J. Duffy \& G. Giuliani (Orgs.). Selected readings in child psychology. Berkeley: McCutchan Publishing Corporation. 
Bennetti, S. P. C. \& Balbinotti, M. A. A. (2002). Inventário de práticas parentais. Manuscrito não publicado. São Leopoldo, RS: Unisinos, Centro de Ciências da Saúde.

Block, J. H., Block, J. \& Morrison, A. (1981). Parental agreement and disagreement on childrearing orientations and gender related personality correlates in children. Child Development, 52, 965-974.

Bronfenbrenner, U. (1996). A ecologia do desenvolvimento humano: experimentos naturais e planejados. Porto Alegre, RS: Artes Médicas.

Carneiro, T. F. (1983). Família: diagnóstico e terapia. Rio de Janeiro: Zahar.

Chao, R. K. (1994). Beyond parental control and authoritarian parenting style: Understanding Chinese parenting through the cultural notion of training. Child Development, 65, 1111-1119.

Chen, X., Liu, M. \& Li, D. (2000). Parental warmth, control, and indulgence and their relations to adjustment in Chinese children. A longitudinal study. Journal of Family Psychology, 14(3), 401-419.

Cicchetti, D. \& Toth, S. L. (1998). The development of depression in children and adolescents. American Psychologist, 53 (2), 221-241.

Collins, W. \& Russell, G. (1991). Mother-child and father-child relationships in middle childhood and adolescence: A developmental analysis. Developmental Review, 11, 99-136.

Costa, F. T., Teixeira, M. A. P. \& Gomes, W. B. (2000). Responsividade e exigência: duas escalas para avaliar estilos parentais. Psicologia Reflexão e Crítica, 13(3), $465-473$.

Darling, N. \& Steinberg, L. (1993). Parenting style as context. An integrative model. Psychological Bulletin, 113 (3), 487-496.

Dekovi, M., Janssens, J. M. A. \& Gerris, J. R. M. (1991). Factor structure and construct validity of the block child rearing practices report (CRPR). Psychological Assessment: A Journal of Consulting and Clinical Psychology, 3(2), 182-187.

Downey, G. \& Coyne, J. (1990). Children of depressed parents: An integrative review. Psychological Bulletin, $108,1,50-76$.

Frosch, C. A. \& Mangelsdorf, S. C. (2001). Marital behavior, parenting behavior, and multiple reports of preschoolers' behavior problems: mediation or moderation? Developmental Psychology, 37 (4), 502-519.

Furman, W. \& Buhrmester, D. (1992). Age and sex differences in perceptions of networks of social relationships. Child Development, 63, 103-115.

Grotevan, H. D. \& Carlson, C. I. (1989). Family Assessment: A guide to methods and measures. New York: The Guilford Press.

Grusec, J., Hastings, P. \& Mammone, N. (1994). Parenting cognition and relationship schemas. Em J. Smetana (Orgs.). Beliefs about parenting: Origins and developmental implications (p. 5-19). San Francisco, CA: Jossey-Bass Publishers.

Holden, G. W. (1995). Parental attitudes towards childrearing. Em M. H. Bornstein (Org.). Handbook of parenting, Vol. 3: Status and social conditions of parenting. (p. 359-392). Hillsdale: Lawrence Erlbaum Associates, Inc.

Holden, G. W., \& Edwards, J. (1989). Parental attitudes toward child rearing: Instruments, issues, and implications. Psychological Bulletin, 106, 29-58.

Holden, G. W., Geffner, R. \& Jourilles, E. N. (Orgs.) (1998). Children exposed to marital violence: Theory, research, and applied issues. Washington: American Psychological Association.

Karpinski, A. \& Hilton, J. L. (2001). Attitudes and the Implicit Association Test. Journal of Personality and Social Psychology, 81(5), 774-788.

Kerns, K. A., Aspelmeier, J. E., Gentzler, A. L. \& Grabill, C. M. (2001). Parent-child attachment and monitoring in middle childhood. Journal of Family Psychology, 15 (1), 69-81.

Lamb, M. E., Pleck, J. H., Charnov, E. L. \& Levine, J. A. (1987). A Biosocial perspective on paternal behavior and involvement. Em J. B. Lancaster, J. Altman, A. Rossi \& L. R. Sherrod (Orgs.). Parenting across the lifespan: Biosocial perspectives (p. 11-42). New York, NY: Academic.

Lovejoy, M. C., Weis, R., O’Hare, E. \& Rubin, E. 1999). Development and initial validation of the parent behavior inventory. Psychological Assessment, 11(4), 534-545.

Luster, T. \& Okagaki, L. (1993). Parenting: An ecological perspective. Hillsdale, NJ: Lawrence Erlbaum Associates Inc.

Nicholas, K. B \& Bieber, S. L. (1996). Parental abusive versus supportive behaviors and their relation to hostility and aggression in young adults. Child Abuse and Neglect, 20 (12), 1195-1211.

Nogueira, Y. (1988). Atitudes maternas: estudo do PARI (Parental Attitude Research Instrument) em amostra brasileira. Arquivos Brasileiros de Psicologia, 40, 48-62.

Psico-USF, v. 8, n. 2, p. 103-113, Jul./Dez. 2003 
Oliveira, E. A., Frizzo, G. B. \& Marin, A. H. (2000). Atitudes maternas diferenciais para com meninos e meninas de quatro e cinco anos. Psicologia Reflexão e Crítica, 13(3), 363-371.

Rosen, K. S. \& Rothbaum, F. (1993). Quality or parental caregiving and security of attachment. Developmental Psychology, 29 (2), 358-367.

Schaeffer, E. S. \& Bell, R. Q. (1958). Development of a parental attitude research instrument. Child Development, 29, 339-361.

Sears, R. R.; Maccoby, E. E. \& Levin, H. (1970). The child rearing process. Em J. Duffy \& G. Giuliani. Selected readings in child psychology. Berkeley, CA: McCutchan Press.

Shelton, K., Frick, P. \& Wooton, J. (1996). Assessment of parenting practices in families of elementary school-age children. Journal of Clinical Child Psychology, 25(3), 317-329.

Sines, J. O., Clarke, W. M. \& Lauer, R. M. (1984). Home Environment Questionnaire. Journal of Abnormal Psychology, 12, 521-529.

Stein, D., Williamson, D. E., Birmaher, B., Brent, D. A., Kaufman, J., Dahl, R. E., Perel, J. M. \& Ryan, N. (2000). Parent-child bonding and family functioning in depressed children and children at high risk and low risk for future depression. Journal American Academy of Child and Adolescence Psychiatry, 39(11), 1387-1395.

Strayhorn, J. \& Weidman, C. (1988). A parent practices scale and its relation to parent and child mental health. Journal American Academy of Child and Adolescence Psychiatry, 27, 613-618.

Super, C. \& Harkness, S. (1996). Parents' cultural belief systems: Their origins, expressions, and consequences. New York, NY: The Guilford Press.

Wamboldt, M. Z. \& Wamboldt, F. (2000). Role of the family in the onset and outcome of childhood disorders: Selected research findings. Journal American
Academy of Child and Adolescence Psychiatry, 39(10), 1212-19.

Wasserman, G., Miller, L., Pinner, E. \& Jaramillo, B. (1996). Parenting predictors of early conduct problems in urban, high-risk boys. Journal American Academy of Child and Adolescence Psychiatry, 35 (9), 1227-36.

Recebido em agosto de 2003 Reformulado em novembro de 2003 Aprovado em dezembro de 2003

\section{Anexo 1- Itens}

1 - Eu tenho conversas amigáveis com meu filho(a).

2 - Eu converso sobre o que acontece na escola.

3 - Eu abraço e beijo meu filho(a).

4 - Eu elogio meu filho(a).

5 - Eu converso sobre assuntos que ela/ele precisa saber sobre a vida.

6 - Eu ensino meu filho(a) as matérias que ele/ela não entende na escola.

7 - Eu converso com ela/ele sobre religião.

8 - Eu ajudo com os deveres escolares.

9 - Eu ajudo com os deveres escolares.

10 - Quando só falar não é suficiente eu dou palmadas no meu filho(a).

11 - As conversas com meu filho(a) terminam em discussões.

12 - É muito difícil para mim fazer com que ele/ela me obedeça.

13 - Eu participo em jogos/atividades com meu filho(a).

14 - Eu levo meu filho(a) para o playground.

15 - Eu vejo TV/escuto música com meu filho (a).

16 - Eu levo meu filho(a) ao cinema.

Sobre os autores:

Silvia Pereira da Cruz Benetti é mestre em Psicologia Educacional, doutora em Psicologia e professora do curso de Psicologia e do curso de especialização em Psicoterapia Psicanalítica da Universidade do Vale do Rio dos Sinos, RS. Atua como supervisora do Programa Interdisciplinar de Promoção e Atenção à Saúde- PIPAS.

Marcos Alencar Abaide Balbinotti é psicólogo, mestre em Psicologia Social e da Personalidade, doutor em Aconselhamento Psicológico e professor na graduação e pós-graduação na Universidade do Vale do Rio dos Sinos e no Programa de Pós-Graduação em Ciências do Movimento Humano, na Universidade Federal do Rio Grande do Sul. 
